\title{
Modifying Flow in the ICA Bifurcation: Pipeline Deployment from the Supraclinoid ICA Extending into the M1 Segment- Clinical and Anatomic Results
}

\author{
E. Nossek, D.J. Chalif, S. Chakraborty, and A. Setton
}

\begin{abstract}
BACKGROUND AND PURPOSE: Utility of the Pipeline Embolization Device extending to the $\mathrm{M} 1$ and its clinical and flow consequences at the ICA bifurcation have not been characterized. We analyzed flow modification in cases where a single Pipeline Embolization Device was deployed from the $\mathrm{Ml}$ to the distal supraclinoid ICA, covering the $\mathrm{Al}$, for aneurysm treatment.
\end{abstract}

MATERIALS AND METHODS: A1 flow modifications and size regression in postprocedure and follow-up angiography were analyzed. Vessel diameters and ratios of the proximal Al and Ml segments and the distal ICA were assessed. Relationships between Pipeline Embolization Device nominal diameter and the vessel diameters at landing zones were obtained. Clinical assessments after flow modification were documented.

RESULTS: Six of 7 patients demonstrated no change of flow in the anterior cerebral artery/anterior communicating artery complex at immediate postembolization angiography. All patients who underwent follow-up angiography demonstrated size regression of the ipsilateral A1. Midterm follow-up angiography revealed complete reversal of flow in the ipsilateral A1 in 4 of 5 patients. One patient did not demonstrate flow modification. This patient had a dominant ipsilateral Al. Vessel ratios in this case demonstrated a unique configuration in favor of maintaining patency of the ipsilateral Al. There were no clinical or radiographic signs of ischemia. One patient experienced asymptomatic angiographic in-stent stenosis at the M1.

CONCLUSIONS: We found that deployment of a Pipeline Embolization Device from the distal supraclinoid ICA to the M1 may result in reversal of flow in the anterior cerebral artery/anterior communicating artery complex and regression of the ipsilateral A1. Preoperative anatomic quantitation and sizing of the Pipeline Embolization Device may predict flow modification results.

ABBREVIATIONS: $\mathrm{ACA}=$ anterior cerebral artery; $\mathrm{AchoA}=$ anterior choroidal artery; AcomA = anterior communicating artery; $\mathrm{FD}=$ flow diverter; ICAb = internal carotid artery bifurcation; OphA = ophthalmic artery; PED = Pipeline Embolization Device

$\mathbf{F}_{\text {a }}$

low-diverter (FD) devices were originally reported to modify and redirect flow as a treatment option for wide-neck aneurysms. ${ }^{1,2}$ Recently, extended use for FDs for more distal and small aneurysms has also been described. ${ }^{3-6}$ The Pipeline Embolization Device (PED; Covidien, Irvine, California) has been approved specifically to treat wide-neck proximal ICA aneurysms at the cavernous and ophthalmic segments. ${ }^{2}$ There are cases, however, where the PED may be used to treat wide-neck aneurysms that are located distal to the ophthalmic segment, at the level of the pos-

Received March 25, 2014; accepted after revision April 21.

From the Department of Neurosurgery, Hofstra North Shore-Long Island Jewish School of Medicine and North Shore-Long Island Jewish Health System, Manhasset, New York.

Please address correspondence to Avi Setton, MD, Department of Neurosurgery, North Shore-Long Island Jewish Health System, North Shore University Hospital, 300 Community Dr, 9 Tower, Manhasset, NY 11030; e-mail: asetton@nshs.edu

三 Indicates article with supplemental on-line table.

http://dx.doi.org/10.3174/ajnr.A4013 terior communicating artery or the anterior choroidal artery (AchoA) segments, as well as those located at the ICA bifurcation (ICAb).

The minimal PED landing length that is recommended to achieve both good anchoring of the stent and efficient flow diversion is approximately $5 \mathrm{~mm}$ proximal and $5 \mathrm{~mm}$ distal to the aneurysmal neck. In cases where the length of the ICA from the distal neck of the aneurysm to the ICA bifurcation is shorter than $5 \mathrm{~mm}$, or alternately, if the treated aneurysm is an ICAb aneurysm, the distal PED should land and be anchored in the proximal M1 segment. Descriptions of PED deployment that cover the A1 segment origin are rare ${ }^{6}$; in addition, flow modification at the level of the ICAb is challenging to predict. In our review of the current literature, we did not find any descriptions of the hemodynamic effects or clinical efficacy of PED deployment in the ICAb extending to the M1 segment.

We described our experience with cases of PED deployment extending into the M1 segment and focused on the anatomic flow 

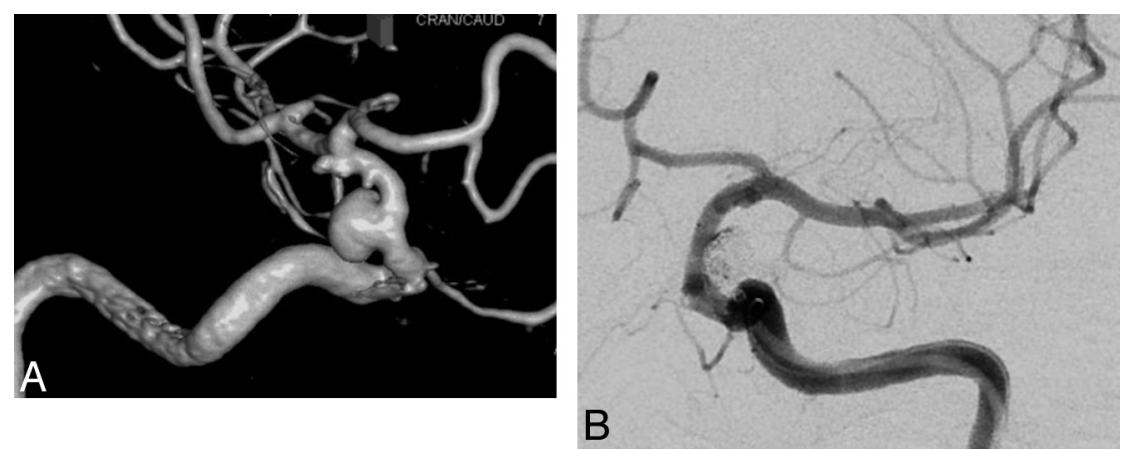

FIG 1. A, A DSA 3D reconstruction of a superior hypophyseal wide-neck aneurysm (1 small additional aneurysm is seen at the AchoA level). $B$, The aneurysm was treated with coil embolization and 1 PED, covering the right Al segment. Postembolization injection demonstrates filling of a large Al segment through the PED.

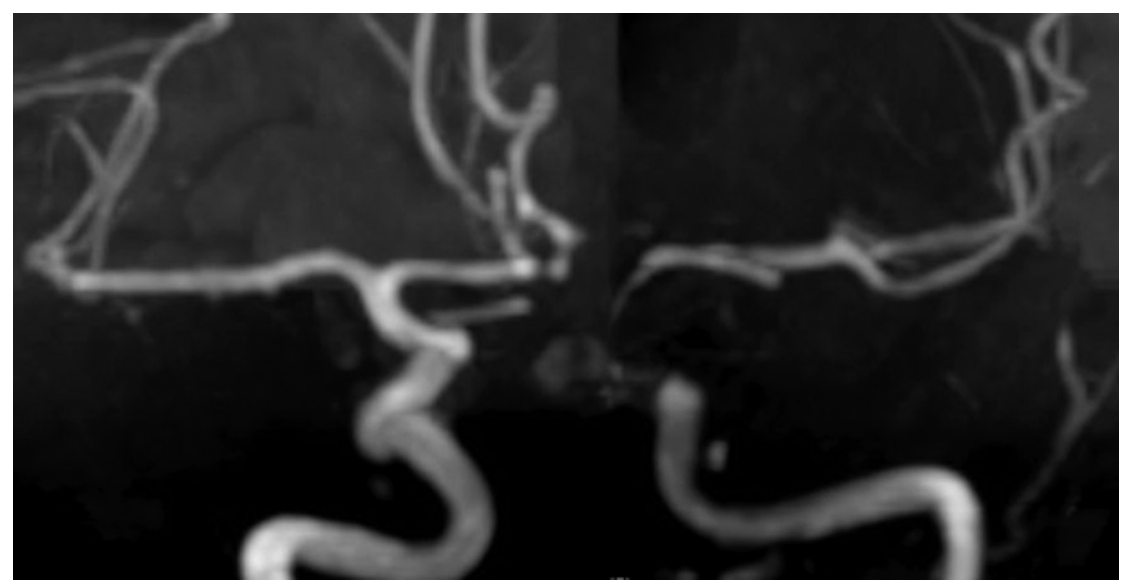

FIG 2. Follow-up TOF MRA at 3 months postprocedure demonstrates regression of the ipsilateral $\mathrm{A} 1$ segment and complete occlusion of the aneurysms.
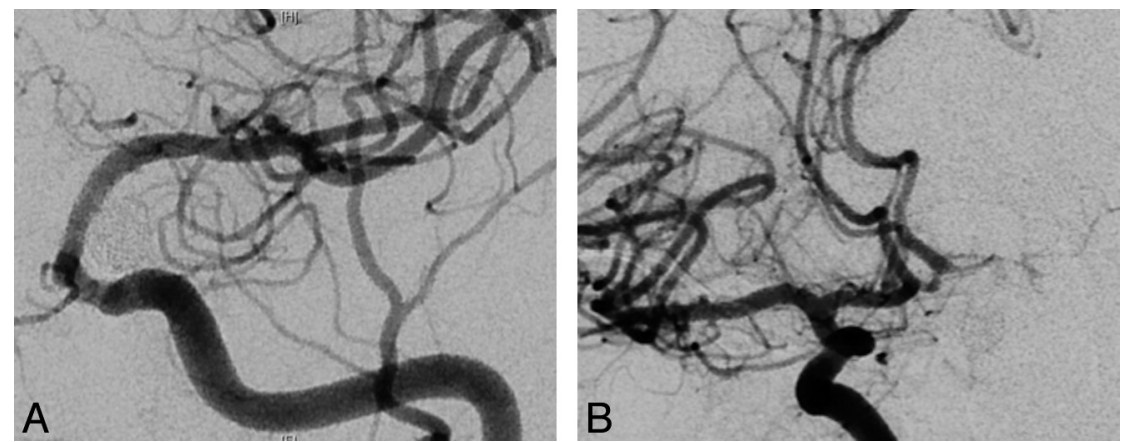

FIG 3. A, Seven-month follow-up angiography demonstrates complete aneurysmal occlusion with no antegrade filling of the ipsilateral Al segment through the flow diverter. $B$, Contralateral ICA injection demonstrates complete reversal of flow and filling of the ipsilateral Al segment perforators. procedure to predict the competence of the collateral circulation (contralateral ICA, bilateral Al segments, and the anterior communicating artery [AcomA]) to supply both anterior cerebral arteries (ACAs). In all cases, a single PED was deployed, covering the ipsilateral A1 segment (Fig 1). In cases where a second PED was used (either for additional aneurysmal neck coverage or for proximal anchoring of the first PED due to lack of proper wall apposition), there was specifically no additional duplicated PED coverage over the origin of the ipsilateral A1 segment or over the AchoA.

Absolute anatomic vessel diameters and ratios between the sizes of the proximal aspects of the A1 segments, M1 segments, and the distal supraclinoid ICA were assessed in calibrated DSA images. Ratios between the PED nominal diameter and the diameters of the proximal M1 segment and ICA at the stent landing zones were obtained. All measurements were evaluated in respect to immediate and long-term flow modifications and size regressions (Fig 2) of the ipsilateral A1 segment documented by MRA (without and with contrast) at 3 months postembolization, and by formal angiography at 6-month follow-up. We describe the flow modification in the ipsilateral A1 segment as follows: 1) no change of flow, 2) antegrade flow retardation, and 3) complete reversal of flow (Fig 3).

Immediate and midterm clinical assessments were also obtained and reviewed.

\section{Preoperative Preparation and Procedure}

All patients were started on dual antiplatelet therapy before procedures. A P2Y12 assay (VerifyNow; Accumetrics, San Diego, California) was obtained in all patients to evaluate and confirm the level of platelet inhibition obtained by the dual antiplatelet regimen.

A 4-vessel diagnostic angiogram was modifications achieved at the ICAb. We have analyzed this technique quantitatively in respect to comparative preoperative anterior circle of Willis angioarchitecture and vessel diameters, and in respect to the specific PED dimensions used.

\section{MATERIALS AND METHODS}

This is a retrospective review of patients who were treated for supraclinoid ICA aneurysms between 2012 and 2013 at our institution (Institutional Review Board approval number 13-303). In this cohort, PEDs were deployed from the proximal M1 segment to the distal supraclinoid ICA. Flow assessments were performed before the performed, inclusive of $3 \mathrm{D}$ reconstructed images. A thorough angiographic assessment of flow dynamics in the anterior circle of Willis, with emphasis on the bilateral ACAs and the AcomA complex was performed. We evaluated the collateral flow from the contralateral A1 segment through the AcomA into both proximal A2 segments, and the retrograde flow, across the midline, into the ipsilateral A1 segment. In cases where the standard angiographic assessment was not conclusive, we used cross-compression of the ipsilateral common carotid artery with concomitant contralateral ICA injection. This allowed preliminary assessment of reversal of flow in the Al segment/AcomA complex. 
Ratios of vessel diameters in the anterior circle of Willis

\begin{tabular}{cccccc}
\hline Patient & $\begin{array}{c}\text { A1 Segment/ } \\
\text { M1 Segment }\end{array}$ & $\begin{array}{c}\text { A1 Segment/ } \\
\text { ICA }\end{array}$ & $\begin{array}{c}\text { M1 Segment/ } \\
\text { ICA }\end{array}$ & $\begin{array}{c}\text { PED/ } \\
\text { M1 Segment }\end{array}$ & $\begin{array}{c}\text { Ipsilateral A1 Segment/ } \\
\text { Contralateral } \\
\text { A1 Segment }\end{array}$ \\
\hline 1 & 0.29 & 0.28 & 0.88 & 1.34 & 0.38 \\
2 & 1.12 & 0.66 & 0.59 & 1.72 & 1.42 \\
3 & 0.5 & 0.38 & 0.76 & 1.3 & 0.79 \\
4 & 0.66 & 0.45 & 0.68 & 1.45 & 0.75 \\
5 & 0.72 & 0.46 & 0.64 & 1.59 & 0.98 \\
6 & 0.76 & 0.55 & 0.73 & 1.36 & 1.44 \\
7 & 0.57 & 0.53 & 0.93 & 1.09 & 0.79 \\
\hline
\end{tabular}

nicating artery aneurysm, which was recoiled during the current procedure, demonstrated complete occlusion on 3-month follow-up MRA. The ICAb aneurysm demonstrated marked size reduction, but did not demonstrate complete occlusion. This patient is pending midterm formal angiographic follow-up.

\section{Flow Modification Results}

Immediate antegrade flow retardation in the ipsilateral A1 segment, post-PED de-
All embolization procedures were performed via standard transfemoral approach under full heparinization using the combination of platinum and hydrogel coils. The dimensions of the Pipeline Embolization Device were chosen for each case after quantitation of the size of the aneurysmal neck, the landing zones length, and both the M1 segment and the ICA diameters. We attempted to match the size of the nominal PED diameter relative to the proximal landing zone in the ICA. Accordingly, the PED was oversized in most cases in respect to the distal landing zone in the M1 segment.

\section{Measurements of the Vessel Diameters}

All vessel measurements were obtained on the original calibrated DSA images. Vessel diameters of the ipsilateral M1 segment and the ICA were obtained from injections before PED deployment at the region of the expected landing sites of the proximal and distal ends of the PED. Measurements of the PED landing length in the M1 segment were defined as the distance from the orifice of the M1 segment at the ICAb to the distal end of the PED. Measurements of the ipsilateral A1 segment and the contralateral A1 segment, the M1 segment, and the supraclinoid ICA were evaluated at a distance of $5 \mathrm{~mm}$ from the ICAb.

\section{RESULTS}

\section{Clinical Results}

We treated 7 patients where a single PED was deployed from the mid- and proximal M1 segment into the supraclinoid ICA (Online Table). In the cohort, 5 patients were women. Median age was 62 years (range 35-72). Four patients were treated for multiple aneurysms. The following aneurysms were treated: 3 posterior communicating artery aneurysms, 3 AchoA artery aneurysms, 4 ICAb aneurysms, and 1 Al segment aneurysm. Clinically, all patients were stable in the immediate postprocedural period and all had no new neurologic deficits. All patients maintained their baseline clinical status. There were no radiographic signs of ischemia. One patient demonstrated asymptomatic angiographic instent stenosis at the M1 segment at 6-month follow-up angiography without neurologic sequelae. All aneurysms, with the exception of 1 fusiform ICAb aneurysm, were completely occluded on follow-up imaging.

The ICAb aneurysm that was not completely occluded on 3-month postprocedure MRA follow-up was a fusiform aneurysm. This patient also had a posterior communicating artery aneurysm that had recanalized after coiling in the acute hemorrhage. The PED was deployed from the M1 segment to the ICA to simultaneously cover and treat both aneurysms. The posterior commu- ployment, was noticed in 1 patient. All other patients did not demonstrate any change of flow in ACA/AcomA complex during immediate postembolization angiography. Six of the 7 patients subsequently demonstrated significant delayed flow modification. All 5 patients who underwent short term (3-4 month) MRA follow-up demonstrated size regression of the ipsilateral Al segment. Five patients underwent midterm follow-up angiography (5.5-12 months). Complete reversal of flow in the ipsilateral A1 segment was noted in 4 of 5 patients. One patient did not demonstrate any flow modification. Two patients are awaiting angiographic follow-up.

\section{Baseline Anatomic Configuration and Flow Modification}

All patients who demonstrated regression of the A1 segment or reversal of flow in the AcomA complex manifested an A1 segment/M1 segment average ratio of 0.58 (range 0.29-0.76) and an A1 segment/ICA average ratio of 0.44 (range $0.28-0.55$ ) (Table). The M1 segment/ICA average ratio in this group was 0.77 (range $0.64-0.93$ ). The entirety of the group, except for 2 patients, demonstrated either equal Al segments or a dominant contralateral A1 segment (ipsilateral A1 segment/contralateral A1 segment, average ratio 0.74 [range $0.38-0.98$ ]).

The only patient who did not demonstrate flow modification, immediately or in follow-up MRA imaging and formal angiography, had a dominant ipsilateral A1 segment (ipsilateral A1 segment/contralateral A1 segment ratio of 1.42). The ratios of the A1 segment/M1 segment (1.12) and the A1 segment/ICA (0.66) were higher in this patient relative to the rest of the patients. Interestingly, the ratio between M1 segment/ICA (0.59) in this patient was the lowest among this group.

\section{Anatomic Configuration in Respect to PED Deployed}

Among the 6 patients who demonstrated flow modification, the partial PED distance extending into the M1 segment from the ICAb measured an average of $7.63 \mathrm{~mm}$ (range 4.95-16.3 $\mathrm{mm}$ ) (Table). The mean nominal PED/M1 segment ratio was found to be oversized at 1.36 (range 1.3-1.59). The patient who did not demonstrate any flow modification had a short length $(0.97 \mathrm{~mm})$ of PED in the M1 segment. In addition, in this case we had the highest oversized PED in respect to the M1 segment diameter at the landing zone (ratio 1.72).

\section{DISCUSSION}

Modification of flow in the ACA-AcomA complex by the technique we propose highlights an important hemodynamic concept that has been previously described in other territories-branch 
vessel and perforator patency is intimately related to flow demand and potential collateralization. ${ }^{3,6-8}$

Flow modification and persistence of flow in adjacent branch vessels using a FD, distinct from the parent vessel itself, have been previously described both in animal model laboratory studies, as well as in clinical case series studies. ${ }^{6,8-11}$ The patency of the ophthalmic artery (OphA) covered by FDs was examined. ${ }^{8}$ In this study, $21 \%$ of the OphAs were occluded, all without visual changes or clinical symptoms. Only 2 patients who demonstrated OphA occlusion demonstrated collateral retrograde flow into the OphA. The flow and vascular contribution of these more distal collateral vessels might be the reason for proximal occlusion of branch vessels such as the OphA when using FDs. Importantly, when there is a positive flow/pressure gradient (or persistent "demand"), critical perforators and end-arteries will remain patent, even if covered by a FD. Conversely, when the demand is not present, flow regression and/or vessel occlusion is predicted. ${ }^{3,8}$

Based on these concepts, the placement of a PED was described recently for the treatment of distal MCA aneurysms. ${ }^{4,6,7}$ Among a series of 26 patients, there were 12 patients who all remained asymptomatic, with reduced flow or occlusion of an MCA branch vessel. Similar to flow modification seen at the OphA region, this phenomenon is most likely dependent on the effectiveness and competency of the regional pial collaterals. ${ }^{6}$ An additional report describes the deployment of a PED in an MCA trunk that did not harbor the aneurysm ("competitive" vessel), thus covering the vessel harboring the aneurysm. In long-term follow-up studies, the parent vessel and aneurysm were occluded with reconstitution of the distal territory by pial leptomeningeal collaterals. ${ }^{7}$ Again, adequate collateralization was noted in tandem with flow reduction or vessel occlusion related to PED placement.

In our study, we observed size regression of the ipsilateral A1 segment compared with the immediate postprocedure angiographic studies, and significant flow modification at the level of the ICAb. A reversal of flow was documented, characterized by retrograde filling of the ipsilateral A1 segment from the contralateral A1 segment through the AcomA, which was consistent with quantitated anatomic configurations (A1 segment/M1 segment average ratio of 0.58 , an A1 segment/ICA average ratio of 0.44 , and ipsilateral A1 segment/contralateral A1 segment average ratio 0.74). Medial lenticulostriate arteries on the ipsilateral A1 segment maintained persistent patent flow dependent on the contralateral A1 segment flow through the AcomA. We noted that in cases where there was reduced demand on the ipsilateral A1 segment with concurrent robust collateral flow from the contralateral A1 segment, the ipsilateral vessel demonstrated early regression. This parallels the observations seen with the OphA and MCA aneurysms and further supports the relationship of vascular "demand" and potential flow regression. ${ }^{3,6-8}$ Our experience with 7 patients demonstrated that ipsilateral A1 regression was not associated with any clinical symptomatology or ischemic lesions manifest on imaging, as the A1 segment perforators will "demand" the flow from the adequate collateralization from the contralateral A1 segment.

We believe that in cases where the contralateral A1 segment or the AcomA are hypoplastic, there is a high demand on the ipsilat- eral A1. Thus, this ipsilateral A1 segment will not regress and will maintain antegrade filling, resembling a perforator with no collateralization covered by a PED. Accordingly, in our cohort, the 1 case that maintained antegrade filling without size regression had an anatomic configuration suggestive of high demand on the ipsilateral A1 segment. Here we noted a dominant ipsilateral A1 segment (ipsilateral A1 segment/contralateral A1 segment ratio of 1.42), and high ratios of the A1 segment/M1 segment and the A1 segment/ICA (1.12 and 0.66, respectively). Several other factors, however, may have played a role in the persistent flow in this scenario. Only a minimal length of PED was deployed in the M1 segment, and therefore the PED might have provided only a limited flow diversion at the level of the ICAb. A second factor that might have impacted on flow diversion at the ICAb is the sizing of the PED in respect to the vessel diameter at the M1 segment's landing zone. In the same patient, we had markedly oversized the PED in respect to the M1 segment diameter, to match the supraclinoid ICA diameter. This differential might have created a higher PED porosity that impacted the flow diversion effect. ${ }^{12}$

In a series recently published of MCA aneurysms treated by FDs, 1 patient had a PED deployment that covered the A1 segment. This patient's course was complicated by a TIA with demonstration of flow attenuation in the A1 segment. Of note, this patient was treated with 2 PEDs and had discontinued antiplatelet therapy. Questionable patency of the ipsilateral Al segment or impingement on the patency of M1 perforators may have been associated with the clinical course in this setting. Thus, we do not cover the A1 segment origin with more than 1 PED to allow for potential adequate flow as per its demand.

\section{Study Limitations}

Our series is limited in size, composed of 7 cases that were treated by the same technique. We presented midterm clinical and angiographic follow-up of the flow modification observed in the ACAAcomA complex. We have attempted to correlate the vessel diameter ratios with the prediction of future modification of flow; this quantitation, however, is a preliminary assessment that will need to be verified by a larger series of patients and longer periods of clinical and imaging follow-up.

\section{CONCLUSIONS}

We found that the deployment of a single PED extending from the supraclinoid ICA to the M1 segment may result in flow modification marked by retrograde filling as well as regression of the ipsilateral A1 segment. Flow modification is usually predictable according to quantitatable anatomic configurations. Deployment of a single PED, when deemed necessary, may be used safely in the treatment of selected aneurysms located in the distal supraclinoid ICA, ICAb, and the A1 and M1 segments.

\section{REFERENCES}

1. Briganti F, Napoli M, Tortora F, et al. Italian multicenter experience with flow-diverter devices for intracranial unruptured aneurysm treatment with periprocedural complications-a retrospective data analysis. Neuroradiology 2012;54:1145-52

2. Becske T, Kallmes DF, Saatci I, et al. Pipeline for uncoilable or failed aneurysms: results from a multicenter clinical trial. Radiology $2013 ; 267: 858-68$ 
3. Saatci I, Yavuz K, Ozer C, et al. Treatment of intracranial aneurysms using the Pipeline flow-diverter embolization device: a single-center experience with long-term follow-up results. AJNR Am J Neuroradiol 2012;33:1436-46

4. Zanaty M, Chalouhi N, Tjoumakaris SI, et al. Flow diversion for complex middle cerebral artery aneurysms. Neuroradiology 2014; 56:381-87

5. Chalouhi N, Starke RM, Yang S, et al. Extending the indications of flow diversion to small, unruptured, saccular aneurysms of the anterior circulation. Stroke 2014;45:54-58

6. Yavuz K, Geyik S, Saatci I, et al. Endovascular treatment of middle cerebral artery aneurysms with flow modification with the use of the Pipeline embolization device. AJNR Am J Neuroradiol 2014;35:529-35

7. Wajnberg E, Silva TS, Johnson AK, et al. Progressive deconstruction: a novel aneurysm treatment using the Pipeline embolization device for competitive flow diversion: case report. Neurosurgery 2014; 10(suppl 1):E161-66
8. Puffer RC, Kallmes DF, Cloft HJ, et al. Patency of the ophthalmic artery after flow diversion treatment of paraclinoid aneurysms. J Neurosurg 2012;116:892-96

9. Darsaut TE, Bing F, Salazkin I, et al. Flow diverters can occlude aneurysms and preserve arterial branches: a new experimental model. AJNR Am J Neuroradiol 2012;33:2004-09

10. Kallmes DF, Ding YH, Dai D, et al. A new endoluminal, flow-disrupting device for treatment of saccular aneurysms. Stroke 2007; 38:2346-52

11. Fiorella D, Woo HH, Albuquerque FC, et al. Definitive reconstruction of circumferential, fusiform intracranial aneurysms with the Pipeline embolization device. Neurosurgery 2008;62:1115-20; discussion 1120-21

12. Shapiro M, Raz E, Becske T, et al. Variable porosity of the Pipeline embolization device in straight and curved vessels: a guide for optimal deployment strategy. AJNR Am J Neuroradiol 2014;35: 727-33 